\title{
Hands-on reconfigurable robotic surgical instrument holder arm
}

\author{
Piyamate Wisanuvej, Konrad Leibrandt, Jindong Liu, and Guang-Zhong Yang
}

\begin{abstract}
The use of conventional surgical tool holders requires an assistant during positioning and adjustment due to the lack of weight compensation. In this paper, we introduce a robotic arm system with hands-on control approach. The robot incorporates a force sensor at the end effector which realises tool weight compensation as well as hands-on manipulation. On the operating table, the required workspace can be tight due to a number of instruments required. There are situations where the surgical tool is at the desired location but the holder arm pose is not ideal due to space constraints or obstacles. Although the arm is a non-redundant robot because of the limited degrees of freedom, the pseudo-null-space inverse kinematics can be used to constrain a particular joint of the robot to a specific angle while the other joints compensate in order to minimise the tool movement. This allows operator to adjust the arm configuration conveniently together with the weight compensation. Experimental results demonstrated that our robotic arm can maintain the tool position during reconfiguration significantly more stably than a conventional one.
\end{abstract}

\section{INTRODUCTION}

Many surgical procedures require tools to hold and maintain instruments or part of the patient body in position while the surgeon performs surgical tasks. In general, the instrument holders are made in the form of an articulated arm where one side is mounted to a fixed foundation. The arm has a control knob or lever to lock the joints in place. Examples of such holder arm include: Martin Arm System (KLS Martin Group, Germany), TEM Instrument Support Arm (Richard Wolf, Germany), Mayfield Head Clamp (Integra LifeSciences, USA). These arms are purely mechanicalbased for ease of construction, robustness, and sterilisability. However, due to the lack of weight compensation, the operator has to carry both the weight of the tool and its payload which makes it difficult to be manoeuvred safely. An assistant is usually required when operating the mechanical holding arms.

Some holder arm systems use electronic locking mechanism to reduce the time required to release and lock the joints. Such systems include Point Setter (Mitaka, Japan), EndoTAIX (SurgiTAIX AG, Germany). Although these devices are more convenient to use due to instant locking/releasing mechanism, they are still not weight compensated. There are several commercial robotic systems for tool

Piyamate Wisanuvej is supported by the Institute of Global Health Innovation, Imperial College London and the Faculty of Engineering, Kasetsart University. Konrad Leibrandt is supported by the Imperial College PhD Scholarship Scheme and EPSRC UK.

All authors are with the Hamlyn Centre for Robotic Surgery, Imperial College London, SW7 2AZ, UK. Piyamate Wisanuvej is also with Kasetsart University, Bangkok, Thailand. \{piyamate.w12, k.leibrandt12, j.liu, g.z.yang\}@imperial.ac.uk

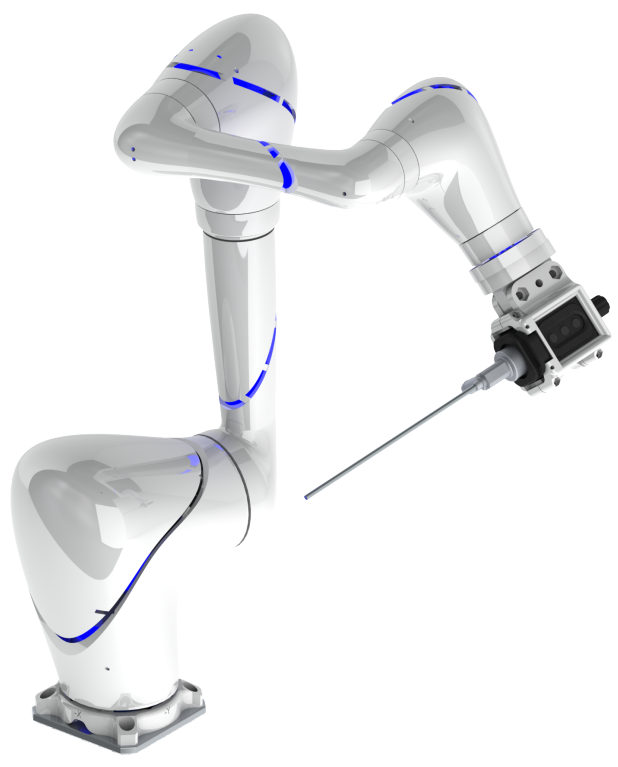

Fig. 1. The Hamlyn Arm with an endoscopic instrument attached.

holding with weight compensation in surgical applications, but they are usually integrated with the instrument system which is not suitable for general purpose usage.

With increasing maturity of compliant robotic arms, they are used extensively in human environments such as healthcare, rehabilitation, surgery, and social humanoid robots [1].

One of the approaches applied to robots in human environment is cooperative manipulation concept where both the robot and the operator manipulate the same tool by physical contact with the robotic arm. It is also termed as "hands-on" manipulation. Such a design has been used in industry to shorten the programming time to certain tasks. For instance, Robotiq proposed "Kinetiq Teaching" technology [2] to quickly program welding trajectories by hand guiding the robotic arm. Rethink robotics designed Baxter robot which can be taught to do pick-and-place task by hand guidance. KUKA and DLR produced the lightweight robot (LWR) arm for collaborative control in which the operator can guide the robot by manipulating the robot arm directly. In other robotic fields, Taylor [3], [4] proposed the "Steady-Hand" robotic system to provide smooth and precise positional control of a hand-held surgical instrument in retinal microsurgery. A hip replacement surgical robot, RobotDoc [5], has been used for positioning surgical tools. Davies proposed a hands-on Acrobot together with ActiveConstriant concept for orthopaedic surgery [6]. Barrett WAM 
[7] robotic arm has been applied in upper limb stroke rehabilitation in which the patient can drag the end effector of the arm to exercise. DLR also proposed MiroSurge system [8] with hands-on endoscopic instruments control capability.

Robotic arms designed to operate in a complex or dynamic environment often have redundant kinematic configurations, i.e. the robot has more degrees of freedom (DOF) than necessary, in order to avoid obstacles [9], [10], prevent overturns [11], or tolerate joint failures [12], etc. Particularly, 7-DOF articulated robot arms have been widely used for manipulating objects in 6-DOF space. One common task for redundant robotic arms is to perform a null-space motion with one joint being kept stationary, particularly to keep the end effector static while allowing configuration changes to comply with the surrounding environment. In some situations, however, the number of joints in a robotic arm are limited to six or lower, such as when joint failure occurs or with limited space. It is an impossible job for these non-redundant robotic arms to perform the same motion as the redundant arms. Nevertheless, it is feasible to have redundant-like motion when the position tolerance is loose.

In this paper, we introduce a robotic positioning arm for surgical instrument holding applications, the Hamlyn Arm, see Fig. 1. This arm addresses common issues found in conventional (purely mechanical) surgical positioning arms which is the lack of weight compensation. Such compensations can make manipulation more convenient and require less effort from the user which is demonstrated in our experiment. In many surgical procedures, there can be multiple surgical instruments occupying the operating table workspace. Occasionally, when the surgical tools are repositioned to a preferred location, the resulting holder arm pose might obstruct the required workspace. In this case, the user has to reposition the holder arm linkages while keeping the surgical tool in place, i.e. reconfiguration. This task can be demanding, hence it is often done with multiple operators. To address this issue, we implement a hands-on reconfiguration technique. This enables user to change the arm body while it automatically maintains the mounted tool in place. Although the Hamlyn Arm is not a redundant robot, the pseudo-null space inverse kinematics (Section III-B) allows user to change the joint position while the robot tries to compensate and keep the end effector movement minimal. Another advantage of having robotic holding system is the capability of sensing of the tool position which enables an integration with imaging or navigation system.

The remainder of this paper is organised as follows. The hardware designs and system integration of the Hamlyn Arm are described in Section II. Then, Section III explains the methods for hands-on manipulation with weight compensation, the pseudo-null space inverse kinematics calculations, and the joint compliant control scheme for hands-on reconfiguration. In Section IV, two experiments involving participants to evaluate our methods are presented. The first experiment evaluates the performance of positioning tasks given the different tool weights, as well as the workload required by the users. This study compares the performance of using the holder arm with and without the weight compensation. The second experiment demonstrates how the Hamlyn Arm performed compared to a conventional tool holder in targeting and reconfiguration tasks.

\section{HAMLYN ARM}

The Hamlyn Arm is an articulated robot with six degrees of freedom. It is actuated by brushless DC motors coupled with harmonic drive gears. On-board controllers perform position, velocity, and current regulation. The arm weights $3.0 \mathrm{~kg}$ and reaches $770 \mathrm{~mm}$ at extended pose. The combination of motors and gears are selected so that the arm can handle a maximum payload of $1.5 \mathrm{~kg}$ with limited speed and acceleration. Each joint can also be backdriven by an operator in case of power loss.

The robot communicates via CANopen bus with a computer system in which we have our control system implemented. The high level control software runs on Linux operating system. It perform several tasks including: motion generation, inverse kinematics, and communication with the motor controllers. Cartesian and joint trajectories generation is done using Reflexxes Motion Libraries [13].

The Hamlyn Arm incorporates an ATI Mini40 force/torque sensor by ATI Industrial Automation (NC, USA) at the end effector. The data is sampled with a DAQ PCIe card by National Instrument (TX, USA) running at $32 \mathrm{kHz}$ sampling rate. The measurements are then filtered with a moving average filter with 100 -sample window size. Fig. 1 shows the Hamlyn Arm with a mounted endoscopic instrument.

\section{METHODS}

\section{A. Hands-on positioning with weight compensation}

Positioning is one of the most common purpose of robotic manipulators where users can command the robot end effector to the desired position and orientation. The hands-on approach allows user to cooperatively guide the robot by hands to the desired location. The robot passively follow the user guidance and holds position when the user releases

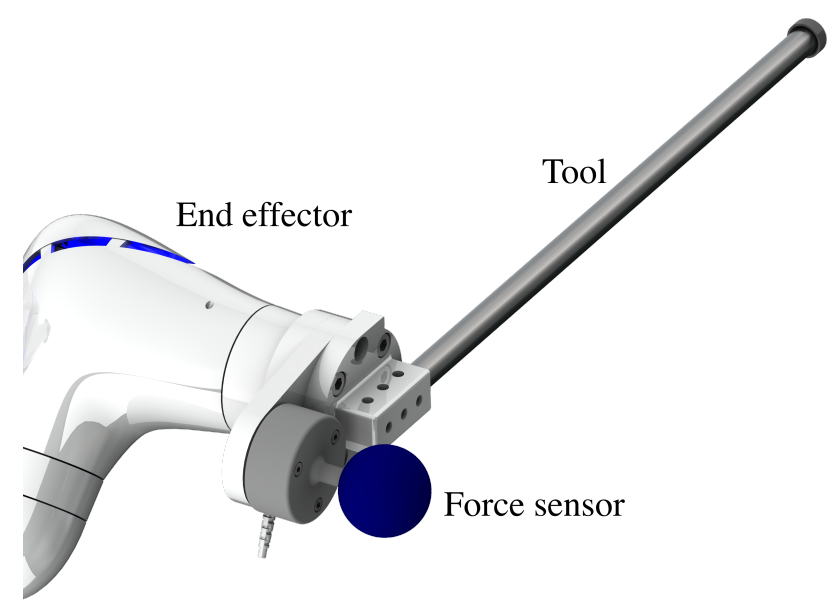

Fig. 2. Hamlyn Arm end effector with a tool and a force sensor mounted 
control. To sense external manipulation forces, a force sensor is installed to the end effector. The force sensor is placed such that the manipulation force is decoupled from the tool's weight. An example of such setup is shown in Fig. 2. The control scheme for hands-on manipulation is implemented by commanding the robotic end effector position according to the force measurements of the sensor. Due to the placement of the sensor, tool's weight and robot's weight do not affect the measurements. Therefore, the user perceive minimal force during manipulation and the tool can maintain position when released. Moreover, this configuration allows tool exchange without required calibration.

Our implementation is as follows. The raw reading from the sensor is subtracted with the baseline force to account for sensor's offset and the weight of the handle. The robot end effector movement is modelled as a virtual mass with a uniform friction in all directions. The force reading $\mathbf{F}$ in Cartesian space is converted to acceleration A by Newton's Law of motion and classical Coulomb friction model [14]. Resulting velocity from an integration of $\mathbf{A}$ is fed into a trajectory generator. The resulting robot pose is sent to the robot as a position command. The inverse kinematics routine then converts the Cartesian space into joint space trajectory for the motor controllers.

\section{B. Pseudo-null-space inverse kinematics}

The standard approach to solve the local inverse kinematics of a redundant robots is to combine the Moore-Penrose pseudoinverse together with a mapping of secondary goals into the null space [15]. Whereas, usually the primary goal is to reach a desired end effector pose $\boldsymbol{T}_{d}$, classical secondary goals include singularity, joint limit, and collision avoidance. Null space mapping allows to use the redundant DoFs to follow secondary goals without impairing the achievement of primary goals. The pseudoinverse matrix $\boldsymbol{J}^{\dagger}$, can be calculated as:

$$
\boldsymbol{J}^{\dagger}=\boldsymbol{V} \operatorname{diag}\left(\frac{1}{\sigma_{1}}, \ldots, \frac{1}{\sigma_{n}}\right) \boldsymbol{U}^{T},
$$

where $\sigma_{i}$ are the singular values of $\boldsymbol{J}$ resulting from singular value decomposition and $\boldsymbol{U}, \boldsymbol{V}$ are the left-singular and rightsingular vectors respectively (i.e. $\boldsymbol{J}=\boldsymbol{U} \operatorname{diag}\left(\sigma_{1}, \ldots, \sigma_{n}\right) \boldsymbol{V}^{T}$ ). $\boldsymbol{J}^{\dagger}$ is further used to calculate the joint velocities of the redundant manipulator;

$$
\dot{\boldsymbol{q}}=\boldsymbol{J}^{\dagger} \dot{\boldsymbol{x}}+\left(\boldsymbol{I}_{n}-\boldsymbol{J}^{\dagger} \boldsymbol{J}\right) \dot{\boldsymbol{q}}_{0}
$$

where $\boldsymbol{I}_{n}$ is the $n$-dimensional identity matrix, and $\dot{\boldsymbol{x}} \in \mathbb{R}^{6}$ the desired end effector velocity to reach the pose $\boldsymbol{T}_{d}$. The second summand of (2) is used to map secondary goals $\dot{\boldsymbol{q}}_{0}$ into the null space. $\dot{\boldsymbol{q}}_{0}$, is defined as:

$$
\dot{\boldsymbol{q}}_{0}=k_{\omega 0}\left(\frac{\partial \omega(\boldsymbol{q})}{\partial \boldsymbol{q}}\right)^{T}
$$

where $k_{\omega 0}$ is a goal weighting factor and $\omega(q)$ is the objective function for the secondary goals.

To overcome the problem of singular robot configurations the damped-least-squares (DLS) inverse $\boldsymbol{J}^{\star}$ can be used at the expense of slower convergence in comparison to the pseudoinverse Jacobian $\boldsymbol{J}^{\dagger}$ :

$$
\boldsymbol{J}^{\star}=\boldsymbol{V} \operatorname{diag}\left(\frac{\sigma_{1}}{\sigma_{1}^{2}+\lambda^{2}}, \ldots, \frac{\sigma_{n}}{\sigma_{n}^{2}+\lambda^{2}}\right) \boldsymbol{U}^{T},
$$

where $\lambda$ is the damping factor. Finally, the robot end effector $\boldsymbol{T}_{e e}$ pose can be calculated using:

$$
\boldsymbol{T}_{e e}=\boldsymbol{f}_{\mathrm{FW}}(\boldsymbol{q}+\dot{\boldsymbol{q}} \Delta t),
$$

where $f_{\mathrm{FW}}$ denotes the closed-form forward kinematic function.

In order to control an underactuated robot with $n$-joints ( $n=5$ in our case) the proposed pseudo-null space concept considers task-space primary goals as $\boldsymbol{x}_{\text {pri }}=\left[t_{x}, t_{y}, t_{z}\right]$ and the remaining three DoFs are considered as secondary goals $\boldsymbol{x}_{\text {sec }}=\left[r_{x}, r_{y}, r_{z}\right]$. Therefore, we split the Jacobian $\boldsymbol{J} \in \mathbb{R}^{6 \times n}$ into $\boldsymbol{J}_{\text {pri }}, \boldsymbol{J}_{\text {sec }} \in \mathbb{R}^{3 \times n}$ with,

$$
\boldsymbol{J}=\left[\boldsymbol{J}_{p r i}^{T}, \boldsymbol{J}_{s e c}^{T}\right]^{T}
$$

as well as the task-space velocities $\dot{\boldsymbol{x}}=\left[\dot{\boldsymbol{x}}_{\text {pri }}^{T}, \dot{\boldsymbol{x}}_{\text {sec }}^{T}\right]^{T}$ and calculate the DLS-inverse $\boldsymbol{J}_{\text {pri }}^{\star}$ and $\boldsymbol{J}_{\text {sec }}^{\star}$, according to (4). Following,

$$
\dot{\boldsymbol{q}}_{0}=f_{k}\left(\Delta \boldsymbol{x}_{p r i}\right) \boldsymbol{J}_{s e c}^{\star} \dot{\boldsymbol{x}}_{s e c}
$$

the secondary goals can be computed, where $f_{k}\left(\Delta \boldsymbol{x}_{p r i}\right)$ is a scalar weighting function depending on the deviation from the primary goals. Combining (2) with (7) where $\boldsymbol{J}_{p r i}^{\star}$ is used instead of $\boldsymbol{J}^{\dagger}$ the joint velocities for the underactuated manipulator can be calculated. Note that using a DLS-inverse results in deviations from the primary goals and furthermore introduces an error into the null-space mapping. The scaling function in (7) is chosen as:

$$
f_{k}=1-\frac{\min \left(\left\|\Delta \boldsymbol{x}_{\text {pri }}\right\|, \Delta x_{\text {pri,max }}\right)}{\Delta x_{\text {pri,max }}},
$$

where $\Delta x_{\text {pri,max }}$ denotes a threshold for the maximum deviation from the primary goals for which secondary goals are considered. For small $\Delta x_{\text {pri,max }}$ secondary goals are quickly ignored and errors in the null-space mapping introduced by the DLS-inverse are mitigated, a large value in contrast represent a looser following of the primary goals.

\section{Hands-on manipulator reconfiguration}

Hands-on positioning the robot via an external force sensor is one way to command the robot cooperatively. However, relying on this alone would constrain the robot in the configuration provided by the inverse kinematics calculation. There are situations where the kinematics solutions are not ideal due to space constraints or obstacles. Using the pseudonull space inverse kinematics can constrain a particular joint of the robot to a specific angle. This can allow the user to change the robot configuration to better suit the space requirements. However, changing this null-space constrain via computer interface might interrupt the user's workflow when performing tasks. Hence, we introduce another handson manipulation mode where user can directly reconfigure 
the robot by pushing its linkages. This is complementary to the hands-on positioning and can be used together to perform manipulation tasks more intuitively.

To implement compliant control on joints, an external joint torque measurement is required. For simplification, our implementation uses motor current measurements provided by the joint controllers. This is a crude measurement which does not take into account the transmission loss and friction. A thresholding on the measured current is used to determine whether a joint is pushed by the user. This threshold is tuned manually to suit a certain range of velocity and acceleration settings. Since the method is oversimplified and inaccurate, the joint output velocity is smoothen by a trajectory generator before being sent to the kinematics calculation.

\section{EXPERIMENTS AND RESULTS}

\section{A. Hands-on positioning study}

An experiment is setup to evaluate hands-on positioning performance on a commercially available robot designed for this purpose using its built-in gravity and payload compensation in comparison to our approach described in section III-A.

The Lightweight Robot 4+ (LWR4+) by KUKA Roboter $\mathrm{GmbH}$ (Augsburg, Germany) is chosen due to its builtin gravity compensation mode. This compensation is done via precise robot modelling and friction compensation by the joint torque sensors. Still, this requires correct payload parameters in order to compensate correctly. In the same time, we can easily equip a force sensor to the end effector and switch the robot operation to position control mode to emulate the Hamlyn Arm. Therefore, it allows experimenting with the same robot hardware for both configurations.

The setup is illustrated in Fig. 3, which consists of a LWR robot and a Mini40 Force/Torque Sensor. Another Lightweight Robot iiwa $14 R 820$ is used to hold the tube

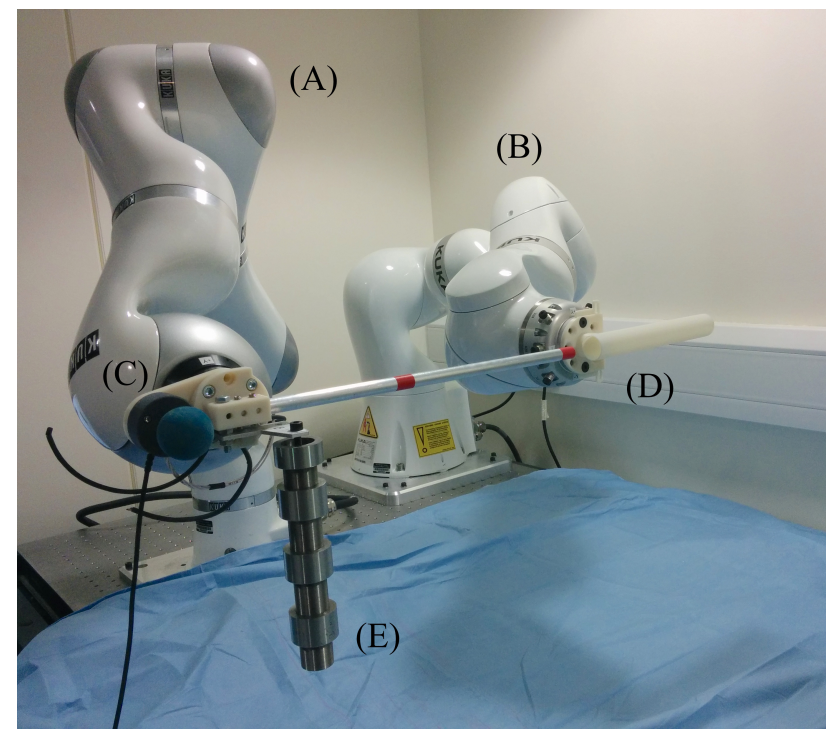

Fig. 3. System components: A) KUKA LWR4+, B) KUKA LBR iiwa, C) ATI Mini40, D) Tool, E) Tube, F) Adjustable weights for the experiments. Dummy tool with variable weights is mounted on the tool to mimic different tool weights.

A tool insertion task is used to validate the hands-on manipulation method. The task for each participant is to pickup and move the tool tip from a starting point far from the tube to the entry point of the tube by holding only the knob with one hand. The tool should be guided along the centreline of the tube until the tooltip reaches the exit point. Participants are asked to perform the task three times, with different tool weights and compensation modes between tasks.

The light and heavy tool are weighted $0.8 \mathrm{~kg}$ and 1.4 $\mathrm{kg}$ respectively. The compensation methods used are the LWR's built-in gravity compensation and our external force sensor based method. Therefore, there are four variations of parameters for this experiment in total. They are denoted as LWR - Light, LWR-Heavy, FT - Light, and FT - Heavy. The task is repeated three times for each participant.

In order to evaluate the effects of manipulating an uncalibrated tool, the LWR has only the correct tool parameters for the light tool. Hence, the user is expected to perceive 0.6 $\mathrm{kg}$ of equivalent tool weight with $L W R-$ Heavy manipulation task.

Each participant is given 10 minutes to get familiar with the system in all task variations. There are total of 5 participants who took part in the experiments, resulting in 60 recorded tasks. Only the manipulation within the tube region is analysed while the rest is discarded. Three performance metrics for each are calculated: completion time, position deviation from the tube centreline, and manipulation force.

\section{B. Hands-on positioning - Results}

Comparison of results from all four tasks with time, force, and position metrics are shown in Table I. We observed only small difference between methods in terms of task completion time, ranging from 4.33 to 5.5 seconds, with $L W R-$ Light being the fastest. Also, it is observed that all the tasks have similar positional deviation, with the FTtasks having slightly smaller deviation. However, there is a significant difference in manipulation force between LWR and FT methods. The force required to move the tool is always under $1.5 \mathrm{~N}$ for the FT methods, while LWR's gravity compensation method requires around $7 \mathrm{~N}$ with calibrated tool and $12 \mathrm{~N}$ with un-calibrated tool as depicted in Fig. 4.

TABLE I

RESULTS FROM HANDS-ON POSITIONING EXPERIMENT SHOWING $M E A N \pm S D$ AND $(M I N-M A X)$

\begin{tabular}{c|c|c|c|c}
\hline & LWR-Light & LWR-Heavy & FT-Light & FT-Heavy \\
\hline Time & $\mathbf{4 . 3 3} \pm 1.94$ & $5.30 \pm 3.06$ & $5.50 \pm 2.20$ & $4.64 \pm 1.14$ \\
$(\mathbf{s})$ & $(2.67-9.47)$ & $(2.25-12.54)$ & $(2.89-8.46)$ & $(3.59-7.33)$ \\
\hline Deviation & $3.89 \pm 1.84$ & $3.83 \pm 1.90$ & $3.39 \pm 1.41$ & $\mathbf{2 . 3 5} \pm 1.31$ \\
$(\mathbf{m m})$ & $(0.14-9.27)$ & $(0.06-9.63)$ & $(0.57-9.11)$ & $(0.15-7.97)$ \\
\hline Force & $7.32 \pm 0.91$ & $11.98 \pm 1.53$ & $\mathbf{1 . 3 6} \pm 0.25$ & $1.46 \pm 0.23$ \\
$(\mathbf{N})$ & $(3.88-11.66)$ & $(6.20-15.28)$ & $(0.44-2.56)$ & $(0.49-2.20)$ \\
\hline
\end{tabular}




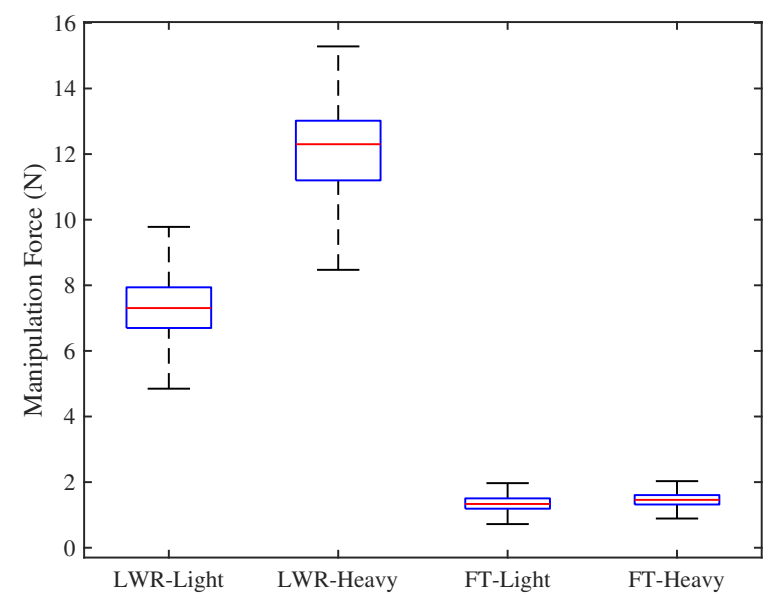

Fig. 4. Comparison of user manipulation force

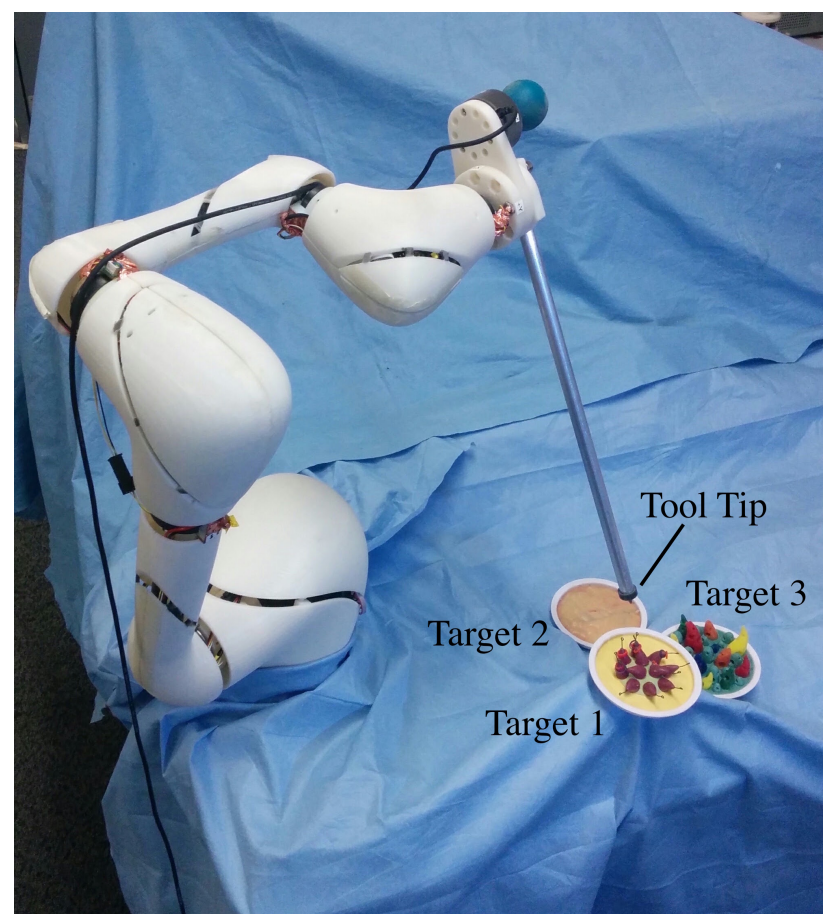

Fig. 5. Experiment setup for targeting and reconfiguration tasks

\section{Hands-on reconfiguration study}

We setup a comparative study to evaluate the benefit of using the compensated robotic tool holder in contrast to manually operated operating table arms when the workspace is limited and a reconfiguration is required. The task chosen is a targeting task where the user is asked to move a long dummy surgical tool from an initial position to three different targets. After reaching the target, the user has to reconfigure the arm base joint to a specific angle in order to keep the workspace around the base joint clear while maintaining the tool position. The experiment setup is shown in Fig. 5.

Tasks are performed using the Hamlyn Arm working in two different modes. A manual mode, where the arm can be locked and released with a foot pedal, emulates the
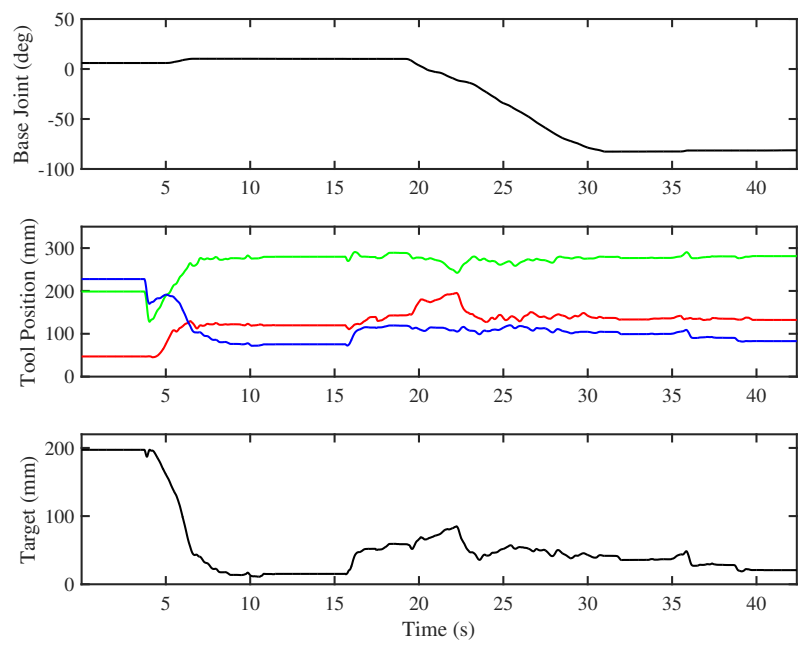

(a) manual mode
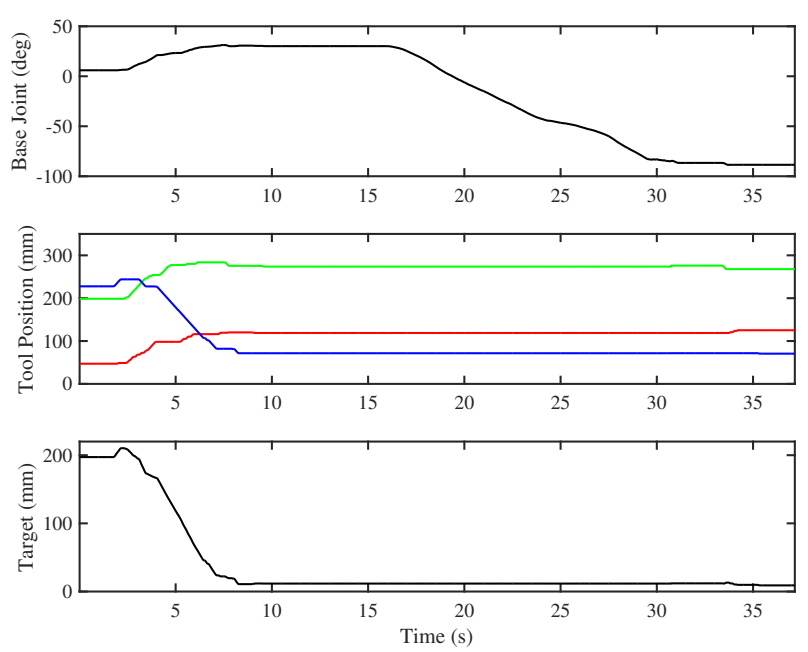

(b) compensated mode

Fig. 6. Examples of collected data from experimental tasks with different operation modes.

functionality of a conventional surgical instrument holder arm. A compensated mode, where the arm exhibits handson positioning and reconfiguration capabilities. All reconfigurations requires the base joint to be moved more than $90^{\circ}$. For each participant, the experiment is done twice with tool weights of $0.8 \mathrm{~kg}$ and $1.4 \mathrm{~kg}$ respectively. Fig. 6 shows examples of collected data where the target position is reached approximately within 8 seconds. Then, the robot is reconfigured. In the compensated case, the robot movement appears smoother and less deviated from the target. Additionally, in the manual mode, a fast drop of the tool position is observed in Fig. 6a at approximately $\mathrm{t}=4$ seconds when the user pressed the release button (red $=\mathrm{X}$, green $=\mathrm{Y}$, blue $=\mathrm{Z}$ ). This is due to the lack of gravity compensation. Similarly to the previous experiment, each participant is given a certain time to learn the system before the actual task. There are 5 participants who performed the experiment. In summary, there are 2 operation modes, 3 targets, 2 tool weights. Therefore, 60 tasks were performed in total. 
TABLE II

RESULTS FROM HANDS-ON RECONFIGURATION EXPERIMENT SHOWING $M E A N \pm S D$ AND $(M I N-M A X)$

\begin{tabular}{c|c|c}
\hline & Manual & Compensated \\
\hline Time & $21.3 \pm 10.5$ & $21.2 \pm 4.8$ \\
$(\mathbf{s})$ & $(5.7-45.2)$ & $(13.0-31.7)$ \\
\hline Maximum & $83.8 \pm 99.1$ & $\mathbf{1 6 . 0} \pm 7.6$ \\
displacement (mm) & $(16.4-394)$ & $(0-32.8)$ \\
\hline Total movement & $805 \pm 406$ & $\mathbf{4 6 . 4} \pm 26.7$ \\
$(\mathbf{m m})$ & $(240-1900)$ & $(0-118)$ \\
\hline
\end{tabular}

(a) maximum target displacement

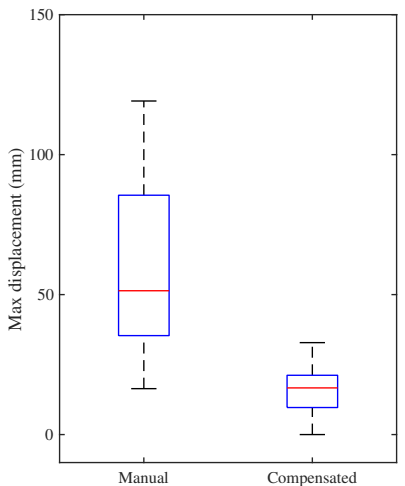

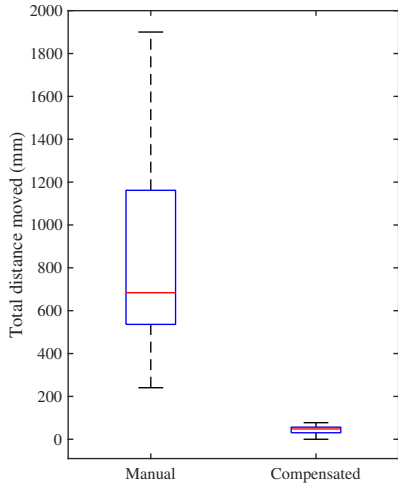

(b) total distance moved
Fig. 7. Results from reconfiguration experiments

\section{Hands-on reconfiguration - Results}

The metrics used to evaluate the performance are: the time used to perform reconfiguration, the maximum displacement from the target during reconfiguration, and the total distance moved during reconfiguration. The quantitative results are listed in Table II. We observed very similar results in the time required which is around 21 seconds. However, the displacement and movement are significantly lower in the compensated mode. The mean of maximum displacement is decreased more than 5 times and the mean of total movement is decreased 17 times with the compensation. Fig. 7 shows the performance improvement of the compensated mode.

\section{COnClusions}

In this paper, we have presented the Hamlyn Arm, a robotic holder arm system that allows hands-on manipulation and reconfiguration based on a tool-mounted force sensor and joint current sensors. The arm is targeted at surgical instrument holding applications where mechanical articulated arms are generally used. Our experimental results show the advantage of using this arm where the weight compensation can reduce the workload of the operator. The placement of the sensor decouples the tool weight from the measurement. Therefore, it allows tool changes during the operation without calibration.

In several surgical procedures, multiple surgical instruments occupy the workspace around the operating table. Another advantage of our system is that the user can reconfigure the holder arm linkages while maintaining the position of the surgical tool. The pseudo-null-space inverse kinematics allows the Hamlyn Arm, which is non-redundant, to perform a reconfiguration while minimising the tool movement with the aid of current sensing-based joint compliant control. Our experiment shows that performing reconfiguration with a purely mechanical holding arm is a difficult task. With our method in the Hamlyn Arm, the amount of tool movement is significantly decreased. In the surgical context, it potentially leads to less damage to the surrounding anatomy.

\section{ACKNOWLEDGEMENT}

The authors would like to thank Petros Giataganas for the CAD renderings and ideas regarding the experiments.

\section{REFERENCES}

[1] M. Hvilshøj, S. Bøgh, O. Skov Nielsen, and O. Madsen, "Autonomous industrial mobile manipulation (AIMM): past, present and future," Industrial Robot: An International Journal, vol. 39, no. 2, pp. 120135, 2012.

[2] N. Lauzier et al., "Force/torque sensor, apparatus and method for robot teaching and operation," US Patent 20150323 398, 2015.

[3] R. Taylor et al., "A steady-hand robotic system for microsurgical augmentation," The International Journal of Robotics Research, vol. 18, no. 12, pp. 1201-1210, 1999.

[4] B. Mitchell et al., "Development and application of a new steadyhand manipulator for retinal surgery," in Proceedings 2007 IEEE International Conference on Robotics and Automation. IEEE, 2007, pp. 623-629.

[5] W. L. Bargar, A. Bauer, and M. Börner, "Primary and Revision Total Hip Replacement Using the Robodoc (R) System." Clinical orthopaedics and related research, vol. 354, pp. 82-91, 1998.

[6] B. Davies et al., "Active-constraint robotics for surgery," Proceedings of the IEEE, vol. 94, no. 9, pp. 1696-1703, 2006.

[7] G. Xu, A. Song, and H. Li, "Control system design for an upperlimb rehabilitation robot," Advanced Robotics, vol. 25, no. 1-2, pp. 229-251, 2011.

[8] R. Konietschke et al., "The DLR MiroSurge-A robotic system for surgery." in Robotics and Automation, 2009. ICRA '09. IEEE International Conference on, vol. 9. IEEE, 2009, pp. 1589-1590.

[9] O. Brock, O. Khatib, and S. Viji, "Task-consistent obstacle avoidance and motion behavior for mobile manipulation," in Robotics and Automation, 2002. Proceedings. ICRA 'O2. IEEE International Conference on, vol. 1. IEEE, 2002, pp. 388-393.

[10] M. Duguleana, F. G. Barbuceanu, A. Teirelbar, and G. Mogan, "Obstacle avoidance of redundant manipulators using neural networks based reinforcement learning," Robotics and Computer-Integrated Manufacturing, vol. 28, no. 2, pp. 132-146, 2012.

[11] Y. Li and Y. Liu, "A new task-consistent overturn prevention algorithm for redundant mobile modular manipulators," in 2005 IEEE/RSJ International Conference on Intelligent Robots and Systems. IEEE, 2005, pp. 418-423.

[12] J. D. English, A. Maciejewski, and Others, "Fault tolerance for kinematically redundant manipulators: Anticipating free-swinging joint failures," Robotics and Automation, IEEE Transactions on, vol. 14, no. 4, pp. 566-575, 1998.

[13] T. Kröger, "Opening the door to new sensor-based robot applicationsThe Reflexxes Motion Libraries," in Robotics and Automation (ICRA), 2011 IEEE International Conference on. IEEE, 2011, pp. 1-4.

[14] H. Olsson, K. Åström, C. Canudas de Wit, M. Gäfvert, and P. Lischinsky, "Friction Models and Friction Compensation," European Journal of Control, vol. 4, no. 3, pp. 176-195, 1998.

[15] B. Siciliano, L. Sciavicco, L. Villani, and G. Oriolo, Robotics: modelling, planning and control. Springer Science \& Business Media, 2010. 